\title{
Production of human milk fat substitute by engineered strains of Yarrowia lipolytica
}

\author{
Govindprasad Bhutada $^{\mathrm{a}, 1}$, Guillaume Menard $^{\mathrm{a}}$, Rupam Kumar Bhunia ${ }^{\mathrm{a}, \mathrm{b}}$, Piotr P. Hapeta ${ }^{\mathrm{c}}$, \\ Rodrigo Ledesma-Amaro ${ }^{\mathrm{c}}$, Peter J. Eastmond ${ }^{\mathrm{a}, \text { " }}$ \\ ${ }^{a}$ Plant Science Department, Rothamsted Research, Harpenden, Hertfordshire, AL5 2JQ, UK \\ ${ }^{\mathrm{b}}$ Plant Tissue Culture and Genetic Engineering, National Agri-Food Biotechnology Institute, Mohali, Punjab, 140306, India \\ ${ }^{c}$ Department of Bioengineering and Imperial College Centre for Synthetic Biology, Imperial College London, London, SW72AZ, UK
}

\section{A R T I C L E I N F O}

\section{Keywords:}

Metabolic engineering

Yarrowia lipolytica

Lipids

$\beta$-Palmitate

OPO

Human milk fat

\begin{abstract}
A B S T R A C T
Human milk fat has a distinctive stereoisomeric structure where palmitic acid is esterified to the middle (sn-2) position on the glycerol backbone of the triacylglycerol and unsaturated fatty acids to the outer (sn-1/3) positions. This configuration allows for more efficient nutrient absorption in the infant gut. However, the fat used in most infant formulas originates from plants, which exclude palmitic acid from the sn-2 position. Oleaginous yeasts provide an alternative source of lipids for human nutrition. However, these yeasts also exclude palmitic acid from the sn-2 position of their triacylglycerol. Here we show that Yarrowia lipolytica can be engineered to produce triacylglycerol with more than $60 \%$ of the palmitic acid in the sn- 2 position, by expression of lysophosphatidic acid acyltransferases with palmitoyl-Coenzyme A specificity. The engineered $Y$. lipolytica strains can be cultured on glycerol, glucose, palm oil or a mixture of substrates, under nitrogen limited condition, to produce triacylglycerol with a fatty acid composition that resembles human milk fat, in terms of the major molecular species (palmitic, oleic and linoleic acids). Culture on palm oil or a mixture of glucose and palm oil produced the highest lipid titre and a triacylglycerol composition that is most similar with human milk fat. Our data show that an oleaginous yeast can be engineered to produce a human milk fat substitute ( $\beta$-palmitate), that could be used as an ingredient in infant formulas.
\end{abstract}

\section{Introduction}

Human milk is the best source of nutrition for infants and it is their main food during the first four to six months of life (Innis 2011; Wei et al., 2019). The lipid fraction provides around half the infant's calories and consists of approximately $98 \%$ triacylglycerol (TAG) (Wei et al., 2019). In human milk fat, palmitic acid (16:0) is esterified to the middle (sn-2) position on the glycerol backbone and oleic acid (18:1) and linoleic acid (18:2) to the outer (sn-1/3) positions (Breckenridge et al., 1969; Christie and Clapperton, 1982; Giuffrida et al., 2019), giving the TAG molecules distinctive stereochemistry that clinical trials have suggested can assist nutrient absorption in the infant gut (Innis 2011; Béghin et al., 2018). This is because sn-1/3 regioselective lipases hydrolyze TAG during digestion to produce fatty acids (FA) and 2-monoacylglycerols (2-MAG). The 2-MAGs are easily absorbed regardless of their acyl moiety. Long chain unsaturated FA are also absorbed, but long chain saturated FA (such as 16:0) tends to form insoluble calcium soaps at intestinal pH and temperature (Innis 2011; Béghin et al., 2018; Wei et al., 2019). The TAG used in most infant formulas is derived from plants (Wei et al., 2019), where $16: 0$ is enriched at the $s n-1 / 3$ positions and excluded from sn-2 (Brockerhoff and Yurkowsk, 1966; Christie et al., 1991). Mixtures of vegetable fats (plus algal and fish oils) can be blended to mimic the fatty acid (FA) composition of human milk, but not its stereoisomeric structure (Wei et al., 2019).

To address this issue, companies have developed a class of 'structured lipids' called human milk fat substitutes (HMFS) (or $\beta$-palmitate) that are produced by enzyme-catalyzed acidolysis (or alcoholysis and esterification) of fractionated vegetable TAG and FAs using sn- $1 / 3$ regioselective lipases (Wei et al., 2019). These HMFS (e.g. Betapol and InFat) currently provide enrichment of 16:0 at the sn-2 position of up to $60 \%$ in the final fat phase of infant formulas. HMFS are relatively costly compared to conventional vegetable fats and it remains technically

\footnotetext{
* Corresponding author. Rothamsted Research, Harpenden, Hertfordshire, AL5 2JQ, UK.

E-mail address: peter.eastmond@rothamsted.ac.uk (P.J. Eastmond).

1 Present address: Nestlé Research, Route du Jorat 57, 1000 Lausanne, Switzerland.
} 
challenging to manufacture a true mimetic at industrial scale that is affordable (Ferreira-Dias and Tecelão, 2014). HMFS ideally requires more than $60 \%$ of the $16: 0$ to be esterified to the sn-2 position in the final fat phase to replicate human milk fat (Breckenridge et al., 1969; López-López et al., 2002). Indeed, the main TAG species in human milk are 1,3-dioleoyl-2-palmitoyl-glycerol (OPO) and 1-oleoyl-2-palmitoyl-3-linoleoyl-glycerol (OPL) or LPO (Giuffrida et al., 2019) and formula makers would likely benefit from an affordable source of pure OPO and OPL that they could then blend with cheaper vegetable fats. A substantial enrichment of 16:0 at the sn-2 position is also found in other animal fats used for human nutrition such as butterfat (Christie and Clapperton, 1982) and lard (Christie and Moore, 1970). Hence, HMFS-type structured lipids also have potential markets in meat and dairy substitute products.

We are investigating whether oleaginous organisms, that normally exclude 16:0 from the sn-2 position of their TAG, can be engineered to produce HMFS. Using the model oilseed plant Arabidopsis thaliana, we have recently showed that it is possible to produce TAG with more than $80 \%$ of the $16: 0$ esterified to the sn- 2 position and where around $40 \%$ of the molecules are OPO (van Erp et al., 2019, 2021). TAG is formed by a cytosolic glycerolipid biosynthetic pathway that's situated on the endoplasmic reticulum (ER) in plants and the enzyme responsible for acylation of the sn-2 position is lysophosphatidic acid acyltransferase (LPAT) (Ohlrogge and Browse, 1995). ER-localized LPAT isoforms discriminate against a 16:0-Coenzyme A (CoA) substrate in plants (Kim et al., 2005). By expressing LPAT isoforms with a preference for 16:0-CoA from other organisms, we were able to drive incorporation of 16:0 into the sn- 2 position of TAG, both in $A$. thaliana with a wild type FA composition (van Erp et al., 2019), and in a multi-mutant with high 16:0 and 18:1 content in its seeds (van Erp et al., 2021).

However, to achieve a sufficient enrichment of 16:0 at sn-2 (above $60 \%$ ) in A. thaliana seed oil, we found that suppression of endogenous LPAT2 expression (Kim et al., 2005) and disruption of phosphatidylcholine:diacylglycerol cholinephosphotransferase (PDCT) (Lu et al., 2009) were also required. PDCT is a plant-specific enzyme that catalyzes head group exchange between diacylglycerol (DAG) and phosphatidylcholine (PC) in A. thaliana seeds (Lu et al., 2009; Bates et al., 2012). PC is the site of acyl-lipid desaturation (Miquel and Browse, 1992) and acyl editing in plants (Stymne and Stobart, 1984; Wang et al., 2012; Bates et al., 2012) and so disruption of PDCT may limit loss of 16:0 from the sn-2 position prior to TAG synthesis (van Erp et al., 2019).

In this study we decided to investigate whether HMFS can be produced by an oleaginous yeast. We chose to use Yarrowia lipolytica as a chassis. Y. lipolytica grows on a variety of carbon sources (Groenewald et al., 2014; Spagnuolo et al., 2018), it is amenable to genetic engineering (GE) and it has a history of use by industry (Groenewald et al., 2014; Ledesma-Amaro and Nicaud, 2016). Y. lipolytica biomass has been defined as a safe novel food by the European Food Standards Agency (Turck et al., 2019) and the US Food and Drug Administration has granted Generally Recognized As Safe status to several products made 'with the assistance of' GE Y. lipolytica strains (Groenewald et al., 2014), including specialty lipids intended for use in human nutrition such as TAG containing omega-3 long chain polyunsaturated FAs (Xue et al., 2013; GRN 000355). Substantial efforts have also been made to improve $Y$. lipolytica lipid productivity using GE (Ledesma-Amaro and Nicaud, 2016) and strains have been reported that achieve cell lipid content, titer, productivity and yield of up to $\sim 70 \%, 99 \mathrm{~g} \mathrm{~L}^{-1}, \sim 1.2 \mathrm{~g}^{-1} \mathrm{~L}^{-1} \mathrm{~h}^{-1}$ and $\sim 0.27 \mathrm{~g} \mathrm{~g}^{-1}$ in nitrogen-limited glucose-fed batch culture (Qiao et al., 2017). The FA composition of $Y$. lipolytica strains cultured on glucose (Carsanba et al., 2020) already resembles that of human milk fat, in terms of the major FA molecular species 16:0, 18:1 and 18:2 (Breckenridge et al., 1969; Giuffrida et al., 2019). However, yeasts (including $Y$. lipolytica) are known to exclude 16:0 from the sn-2 position of their TAG (Thorpe and Ratledge 1972) and so reversing this selectivity is a key step to producing a HMFS in these oleaginous organisms.

\section{Materials and methods}

\subsection{Material and growth conditions}

The $Y$. lipolytica strains used in this study are listed in Table 1 . The media and growth conditions for Escherichia coli and Y. lipolytica have been described by Sambrook and Russell (2001) and Barth and Gaillardin (1996), respectively. Y. lipolytica shake flask cultures were grown in nitrogen limited minimal media as described previously (Bhutada et al., 2017), except that palm oil emulsified with $1 \%$ (v/v) Tween 80 was used as a carbon source in some experiments. For the growth of ura3s or leu $2 \Delta$ auxotrophic strains, media were supplemented with 0.1 $\mathrm{g} \mathrm{L}^{-1}$ uracil or leucine. Bioreactor cultivations were carried out in $1 \mathrm{~L}$ BIOSTAT Qplus fermenters (Sartorius Stedim Biotech $\mathrm{GmbH}$ ) under the following conditions: working volume of $0.5 \mathrm{~L}$, temperature $28{ }^{\circ} \mathrm{C}$, stirring rate $10 \mathrm{~Hz}(600 \mathrm{rpm})$ and aeration $0.6 \mathrm{vvm}$ (vessel volume per minute). $\mathrm{pH}$ was automatically maintained at $\mathrm{pH} 5.7$ by addition of $40 \%$ $(\mathrm{w} / \mathrm{v}) \mathrm{NaOH}$. The cultivation medium contained $25 \mathrm{~g}$ glucose, $25 \mathrm{~g}$ palm oil, $1.26 \mathrm{~g}\left(\mathrm{NH}_{4}\right)_{2} \mathrm{SO}_{4}, 3.0 \mathrm{~g} \mathrm{KH}_{2} \mathrm{PO}_{4}, 0.5 \mathrm{~g} \mathrm{MgSO}_{4} \cdot 7 \mathrm{H}_{2} \mathrm{O}, 0.2 \mathrm{~g}$ leucine, 1 $\mathrm{mL}$ trace metal solution (TMS) and $1 \mathrm{~mL}$ vitamin solution (VS; Hong and Nielsen, 2013) in $1 \mathrm{~L}$ distilled water. The precultures were carried out in the same medium buffered at $\mathrm{pH} 5.7$ with 2-(N-morpholino)ethanesulfonic acid (MES) at $28{ }^{\circ} \mathrm{C}$ on a rotary shaker with a stirring rate of 5 $\mathrm{Hz}(300 \mathrm{rpm})$ for $24 \mathrm{~h}$. Glucose, leucine, TMS and VS solutions were filter-sterilized on $0.22 \mu \mathrm{m}$ membranes. Prior to inoculation, the biomass was washed three times with sterile distilled water. The initial $\mathrm{OD}_{600}$ was set to 0.5 . The samples were taken every $24 \mathrm{~h}$. The experiments were performed in three biological replicates. All PCR reactions for cloning and amplification of sequencing templates were performed using Herculase II Fusion DNA Polymerase (Agilent Technologies), and using GoTaq (Promega) for confirmation of chromosomal integration of the transformation cassettes. The restriction enzymes used in this study were obtained from Roche or New England Biolabs (NEB). The DNA fragments from PCR and restriction digestion were recovered from agarose gels using GeneJET kits (Thermo Scientific). For ligations, the Fast-Link DNA Ligation Kit (Epicenter) or Gibson assembly (Gibson et al., 2009) was used. For transformation into $Y$. lipolytica standard protocols for lithium acetate were used (Le Dall et al., 1994). All plasmids and PCR primers are listed in Supplementary Table 1.

\subsection{Cloning and transformation}

To obtain Y. lipolytica strains expressing eukaryotic LPATs with a substrate preference of 16:0-CoA, we synthesised codon optimised versions of Brassica napus mLPAT1 (van Erp et al., 2019, 2021), Homo

Table 1

$Y$. lipolytica strains used in this study.

\begin{tabular}{|c|c|c|}
\hline Strain & Genotype & $\begin{array}{l}\text { Source/ } \\
\text { description }\end{array}$ \\
\hline WT (W29) & MATa wild type & $\begin{array}{l}\text { Barth and } \\
\text { Gaillardin (1996) }\end{array}$ \\
\hline Po1d & MATa ura3-302, xpr2-322, pURA3-SUC2 & $\begin{array}{l}\text { Barth and } \\
\text { Gaillardin (1996) }\end{array}$ \\
\hline obese & $\begin{array}{l}\text { MATa ura3-302, leu2-270, xpr2-322, pURA3- } \\
\text { SUC2, pox1-6A, tgl4 }, \text {, pTEF-GPD1, pTEF- } \\
\text { DGA2 }\end{array}$ & Lazar et al. (2014) \\
\hline gsy1s & $\begin{array}{l}\text { MATa ura3-302, xpr2-322, pURA3-SUC2, } \\
\text { gsy1A::URA3 }\end{array}$ & This work \\
\hline $\begin{array}{l}g s y 1 \Delta- \\
\quad m L P A T 1\end{array}$ & $\begin{array}{l}\text { MATa ura3-302, xpr2-322, pURA3-SUC2, } \\
\text { gsy1A::YLTEF1 }{ }^{P}-\text { mLPAT1-Syn }{ }^{T}-U R A 3\end{array}$ & This work \\
\hline $\begin{array}{l}g S y 1 \Delta- \\
\quad A G P A T 1\end{array}$ & $\begin{array}{l}\text { MATa ura3-302, xpr2-322, pURA3-SUC2, } \\
\text { gsy1A::YlTEF1 }{ }^{P} \text {-AGPAT1-Syn }{ }^{T} \text {-URA3 }\end{array}$ & This work \\
\hline $\begin{array}{l}\text { gsy14- } \\
\text { LPAAT2 }\end{array}$ & $\begin{array}{l}\text { MATa ura3-302, xpr2-322, pURA3-SUC2, } \\
\text { gsy1 }:: \text { YlTEF }^{P} \text {-LPAAT2-Syn }{ }^{T} \text {-URA3 }\end{array}$ & This work \\
\hline $\begin{array}{l}\text { obese-gsy } 1 \Delta- \\
\text { LPAAT2 }\end{array}$ & $\begin{array}{l}\text { MATa ura3-302 leu2-270 xpr2-322, pURA3- } \\
\text { SUC2, pox1-6A, tgl4A, pTEF-GPD1, pTEF- } \\
\text { DGA2, gsy1 }:: Y l T E F 1^{P} \text {-LPAAT2-Syn }{ }^{T}-U R A 3\end{array}$ & This work \\
\hline
\end{tabular}


sapiens AGPAT1 (van Erp et al., 2021) and Chlamydomonas reinhardtii LPAAT2 (Kim et al., 2018) together with the Tsynt25 $\left(\mathrm{Syn}^{\mathrm{T}}\right)$ synthetic terminator fragment (Curran et al., 2015) (Supplementary Fig. 1). We then linearised the plasmid pGMKGSY_12 (Bhutada et al., 2017), which harbours a glycogen storage elimination cassette flanked by $\sim 1 \mathrm{~kb}$ recombination regions for the glycogen synthase (GSY1) locus, with HindIII. We PCR amplified the strong constitutive TEF1 promoter from $Y$. lipolytica W29 genomic DNA using the primers pair TEF-GSY-F and TEF-GSY-R and assembled the fragments by Gibson assembly to produce the plasmid pGSYTEF. The codon optimised LPAT-Syn ${ }^{\mathrm{T}}$ fragments were excised from pUC7 using HindIII. pGSYTEF was digested with the same enzyme to linearize the vector and it was re-ligated with the gel-purified LPAT-SynT fragments to produce $p$ TEF-mLPAT1, pTEF-AGPAT1 and pTEF-LPAAT2. The correct assembly of the episomal YlGSY1 ${ }^{P}$-loxP-URA3-loxP-TEF ${ }^{P}$ mLPAT1Syn ${ }^{T}-Y l G S Y 1^{T}, \quad Y l G S Y 1^{P}$-loxP-URA3-loxP-TEF ${ }^{P} \mathrm{~A}$ GPAT1Syn $^{T}-Y l G S Y 1^{T}$ and $Y l G S Y 1^{P}$-loxP-URA3-loxP-TEF ${ }^{P}$ LPAAT2Sy $\mathrm{n}^{\mathrm{T}}-$ YlGSY $1^{T}$ cassette was confirmed by sequencing. These cassettes were excised with NotI, gel-purified and used for transformation of strain Po1d or obese (Table 1). Transformants with integration of the cassette at the GSY1 locus were identified by Lugol's iodine staining (1\% KI, 0.5\% $\mathrm{I}_{2}$ ) and confirmed by PCR amplification of genomic DNA flanking the integration site using primer $\mathrm{GSY}^{\mathrm{P}}-\mathrm{F}$ with mLPAT1-R, AGPA1-R, LPAAT2-R or GSY1 ${ }^{\mathrm{T}}-\mathrm{R}$ and by sequencing the products.

\subsection{Metabolite analysis}

Lipids were extracted from $Y$. lipolytica cell pellets as described by (Bhutada et al., 2017), following the methods of Hanscho et al. (2012). TAG was purified from the lipids and regiochemical analysis was performed by lipase digestion following the methods described by (van Erp et al., 2019). Lipid extracts, TAG and 2-MAG fractions were trans-methylated and their FA content were quantified by gas chromatography (GC) coupled to flame ionization detection (FID), as described previously (van Erp et al., 2019), using a 7890A GC system fitted with DB-23 columns ( $30 \mathrm{~m}$ x $0.25 \mathrm{~mm}$ i.d. x $0.25 \mu \mathrm{m}$ ) (Agilent Technologies). Tripentadecanoin was added to the fractions prior to transmethylation to provide an internal standard for quantification. The total FA content of freeze-dried cell pellets and culture medium supernatants were also determined by direct transmethylation and GC-FID (Niehus et al., 2018). Glucose and citrate in the supernatants were identified and quantified by high-performance liquid chromatography (HPLC) using an Aminex HPX87H column (Biorad) coupled to UV and refractive index (RI) detectors (Niehus et al., 2018).

\subsection{Dry biomass determination}

To determine dry biomass, the cell pellets from culture samples were either washed twice with distilled water, filtered on $0.45 \mu \mathrm{m}$ nitrocellulose filters, dried at $97{ }^{\circ} \mathrm{C}$ overnight and weighed (Bhutada et al., 2017) or they were freeze-dried and weighed (Niehus et al., 2018).

\subsection{Statistical analyses}

All experiments were carried out using three or more biological replicates and the data are presented as the mean values \pm standard deviation of the mean (SD). For statistical analysis we either used oneway analysis of variance (ANOVA) with post-hoc Tukey HSD (Honestly Significant Difference) tests, or two-tailed Student's t-tests.

\section{Results}

\subsection{LPAT expression in Y. lipolytica produces TAG with over $60 \%$ of 16:0 at the sn-2 position}

In yeasts, TAG is formed by a glycerolipid biosynthetic pathway whose enzymes are situated on the ER and on lipid droplets (LD)
(Bredeweg et al., 2017; Klug and Daum, 2014) and LPATs are responsible for acylation of the sn-2 position (Benghezal et al., 2007; Jain et al., 2007; Ayciriex et al., 2012, Fig. 1). To determine whether heterologous expression of LPATs with specificity for 16:0-CoA can drive incorporation of 16:0 into the sn-2 position of TAG in $Y$. lipolytica, we selected three different LPATs of eukaryotic origin to test; LPAT1 from B. napus (Bourgis et al., 1999), AGPAT1 from H. sapiens (Agarwal et al., 2011) and LPAAT2 from C. reinhardtii (Kim et al., 2018). All three LPATs have been reported to be capable of using 16:0-CoA as a substrate (Bourgis et al., 1999; Agarwal et al., 2011; Kim et al., 2018). AGPAT1 and LPAAT2 are localized to the ER (Agarwal et al., 2011; Kim et al., 2018), whereas LPAT1 is situated in the chloroplast inner membrane (Bourgis et al., 1999). However, we have shown that expression of a modified version of LPAT1 (mLPAT1) that lacks the N-terminal chloroplast transit sequence results in ER localisation in plants (van Erp et al., 2019).

We synthesised mLPAT1, AGPAT1 and LPAAT2 cDNAs that are codon optimised for expression in $Y$. lipolytica. We then cloned them downstream of the native translational elongation factor EF-1 alpha (TEF1) promoter and integrated the cassettes at the glycogen synthase (GSY1) locus (Bhutada et al., 2017) in strain Po1d. Up to 40 Ura + colonies were selected, screened for glycogen deficiency and cassette integration at the GSY1 locus was confirmed by PCR on genomic DNA and sequencing.

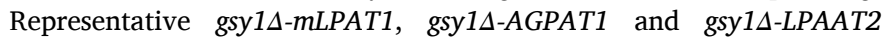
strains, together with wildtype strain W29 (WT) and gsy1 controls, were grown in triplicate in shake flask cultures in nitrogen-limited media with $20 \mathrm{~g} \mathrm{~L}^{-1}$ glycerol as the sole carbon source for $72 \mathrm{~h}$ (Bhutada et al., 2017). Lipids were extracted from the cells (Bhutada et al., 2017) and the total FA and sn-2 FA composition of TAG were analysed (van Erp et al., 2019).

WT cells contained TAG with around 21\% 16:0, 46\% 18:1 and 14\% 18:2 (Fig. 2A and C, Supplementary Table 2). Palmitoleic acid (16:1) and stearic acid (18:0) were also present at $\sim 6 \%$ and $\sim 12 \%$, respectively (Fig. 2C, Supplementary Table 2). As has been reported previously (Thorpe and Ratledge 1972), 16:0 (and 18:0) are virtually absent from the sn-2 position (Fig. 2B and D, Supplementary Table 2). Only $\sim 3 \%$ of the 16:0 in TAG is present at the sn-2 position. Since we chose to integrate cassettes at the GSY1 locus, we also analysed gsy1 cells as a control. The FA composition of TAG from gsy $1 \Delta$ is altered, with a significant $(\mathrm{P}<0.05)$ decrease in the percentage of 16:0 (Fig. 2A), which has been reported previously (Bhutada et al., 2017). However, 16:0 was still excluded from the sn-2 position of TAG in gsy $1 \Delta$ cells (Fig. 2B). Cells of gsy1 $1 \Delta$-mLAT1 exhibited only small differences in the FA composition of TAG verses gsy $1 \Delta$ (Supplementary Table 2) and a small but significant $(\mathrm{P}<0.05$ ) increase in the percentage of 16:0 at sn-2 to $\sim 12 \%$ (Fig. $2 \mathrm{~B}$ ). By contrast, gsy1 $1 \Delta$-AGPAT1 and gsy1 $1-L P A A T 2$ cells contained TAG with both an increase in total 16:0 verses gsy1 1 (Fig. 2A) and a large increase in 16:0 at the sn-2 position ( $\mathrm{P}<0.05$ ) (Fig. $2 \mathrm{~B}$ ). By comparing the total FA composition of TAG to that at sn-2, we calculated that in gsy1$\Delta$-AGPAT1 and gsy $1 \Delta$-LPAAT2 cells respectively, $\sim 58 \%$ and $\sim 63 \%$ of the total 16:0 in TAG is at the sn-2 position (Fig. $2 \mathrm{~B}$ and D). Of the strains we tested, gsy1 $\triangle$-LPAAT2 has a FA composition that is closest to human milk fat (López-López et al., 2002) with $\sim 19 \%$ 16:0, 3\% 16:1, 13\% $18: 0, \sim 50 \% 18: 1$ and $\sim 15 \% 18: 2$ (Fig. 2 C), with more than $60 \%$ of the total 16:0 in TAG at the sn-2 position (Figs. 2B) and 16:0 accounting for nearly $40 \%$ of all the FA at the sn-2 position (Fig. 2D).

\subsection{Culture on palm oil or a mixture of glucose and palm oil improves TAG composition}

$Y$. lipolytica can utilise a range of carbon sources in addition to glycerol, including various sugars and lipids (Spagnuolo et al., 2018). It is known that the carbon source can influence both the quantity of TAG that is produced and its FA composition in Y. lipolytica (Papanikolaou et al., 2003; Athenstaedt et al., 2006; Beopoulos et al., 2008; Vasiliadoua et al., 2018a, b). We therefore grew WT and gsy $1 \Delta-L P A A T 2$ in shake flask culture for $72 \mathrm{~h}$ in nitrogen-limited media with $20 \mathrm{~g} \mathrm{~L}^{-1}$ glucose or $20 \mathrm{~g}$ 


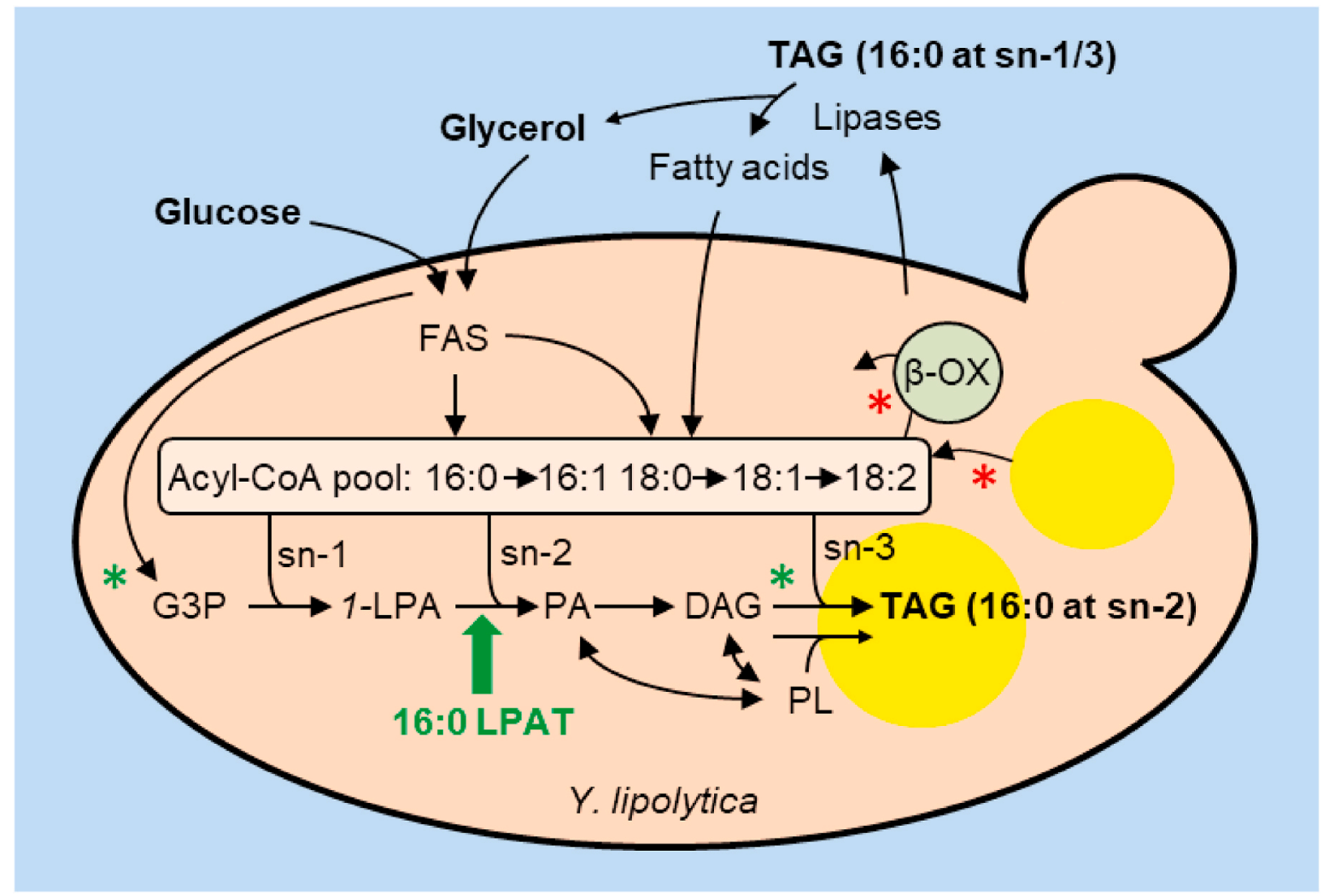

Fig. 1. A diagram illustrating the strategy used in this study to produce HMFS in Y. lipolytica, using glycerol, glucose and/or palm oil as substrates. Heterologous expression of an LPAT with 16:0-CoA specificity (green arrow) was used to enable 16:0 to be esterified to the sn-2 position of 1-LPA and the product PA is then metabolised to TAG. Green and red asterisks mark modifications in an obese strain (Lazar et al., 2014) used to increase lipid accumulation. FAS, fatty acid synthase; $\beta$-OX, peroxisomal FA $\beta$-oxidation; 16:0, palmitic acid; 16:1, palmitoleic acid; 18:0 stearic acid, 18:1, oleic acid; 18:2, linoleic acid; CoA, Coenzyme A; G3P, glycerol-3-phosphate; 1-LPA, sn-1 lysophosphatidic acid; PA, phosphatidic acid, DAG, diacylglycerol, TAG, triacylglycerol; PL, major phospholipids. (For interpretation of the references to colour in this figure legend, the reader is referred to the Web version of this article.)

Glycerol
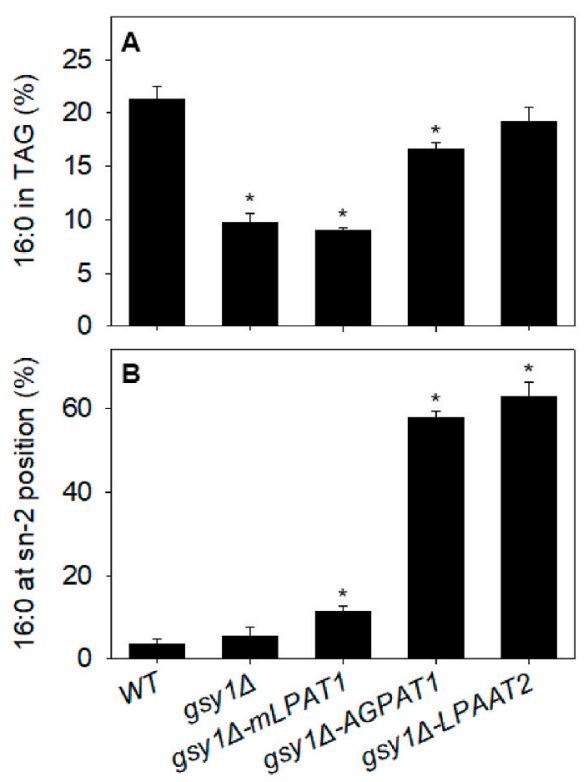

Glycerol
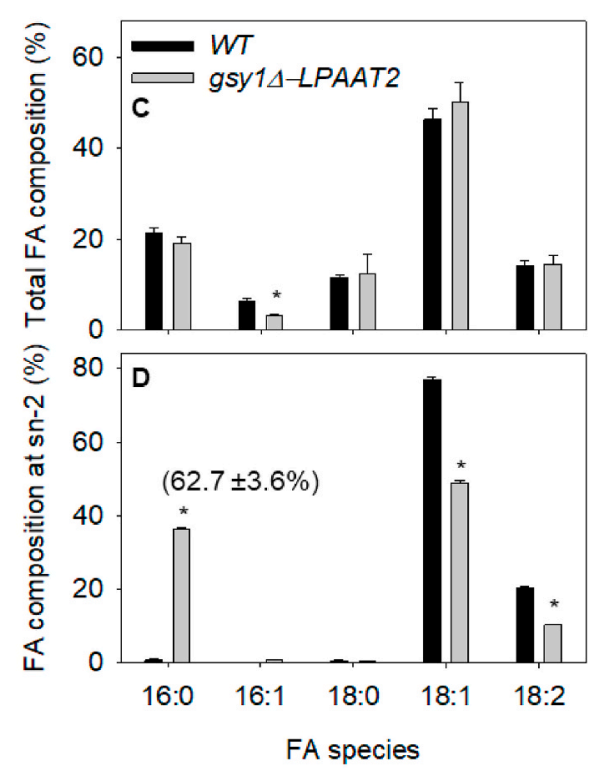

Fig. 2. FA composition of TAG from $Y$. lipolytica strains expressing 16:0-CoA LPATs, cultured on $20 \mathrm{~g}$ $\mathrm{L}^{-1}$ glycerol in nitrogen-limited media. (A) Percentage of 16:0 in TAG. (B) Percentage of 16:0 at sn-2 position. (C) Total FA composition of TAG, (D) FA composition at sn-2 position. The values are means \pm $\mathrm{SD}$ of measurements made on three separate cultures for each genotype. * denote values significantly $(\mathrm{P}<$ 0.05) different from WT (A \& B ANOVA + Tukey HSD test, $C \&$ D Student's t-test). In D the value in brackets is the percentage of $16: 0$ at the sn-2 position.
$\mathrm{L}^{-1}$ palm oil as sole carbon sources, or with a mixture of $10 \mathrm{~g} \mathrm{~L}^{-1}$ of each substrate. We extracted lipids from cells at stationary phase (Bhutada et al., 2017) and determined the total FA and sn-2 composition of TAG (van Erp et al., 2019). The FA composition of TAG from WT and gsy1 $\triangle$-LPAAT2 cells grown on glucose (Fig. 3A) was similar with that of cells grown on glycerol (Fig. 2C) except that 18:0 levels were significantly $(\mathrm{P}<0.05)$ lower $(\sim 8 \%$ versus $\sim 12 \%)$. The percentage of total $16: 0$ that is at the sn- 2 position of TAG was only $\sim 1 \%$ in WT, whereas it 
Glucose
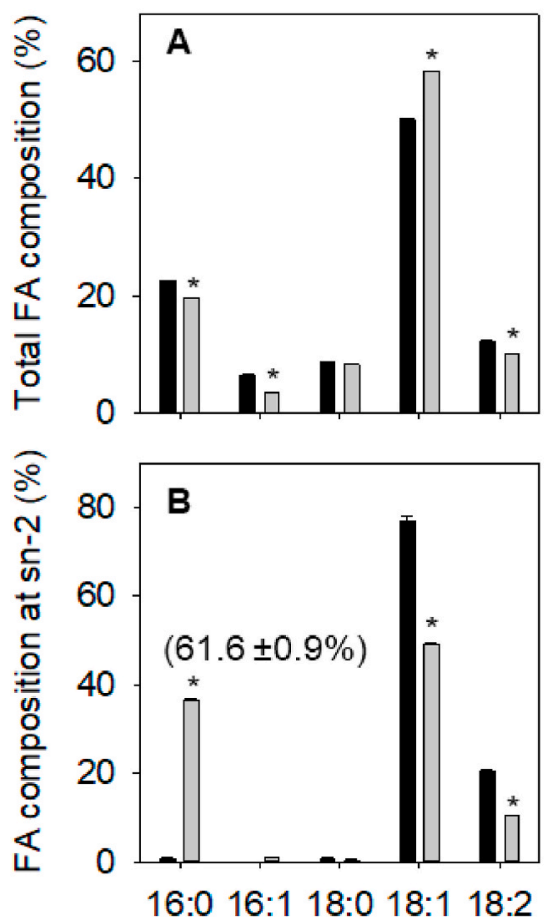

FA species
Palm oil
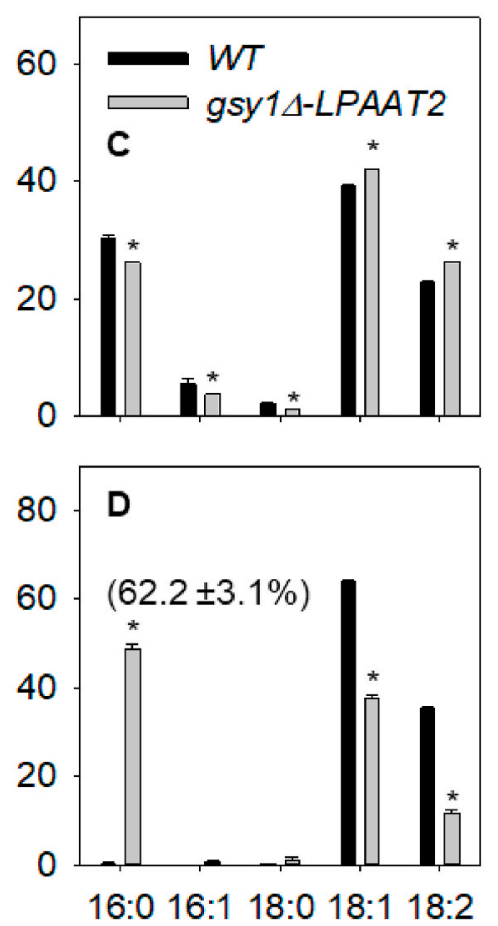

FA species
Glucose + Palm oil
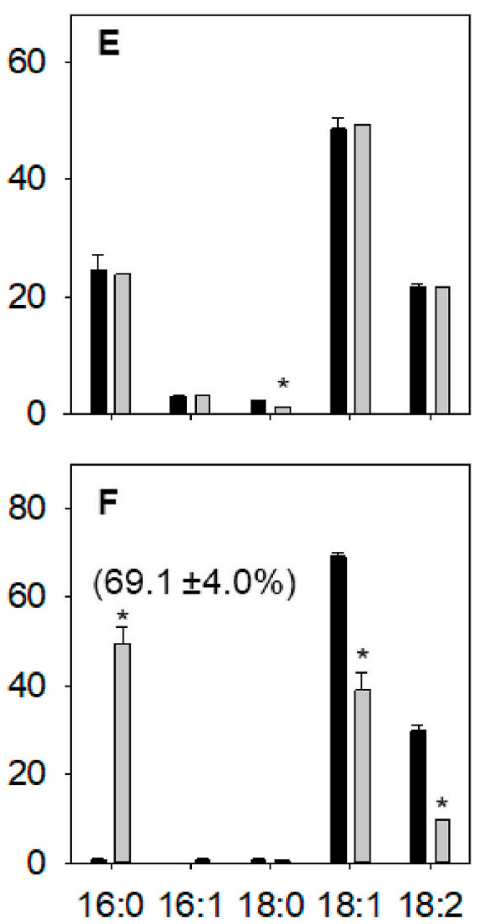

FA species

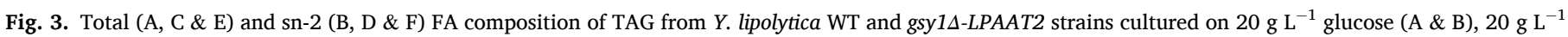

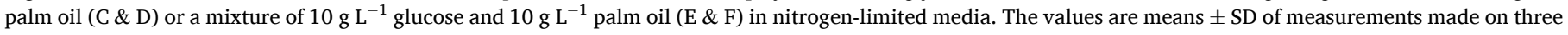

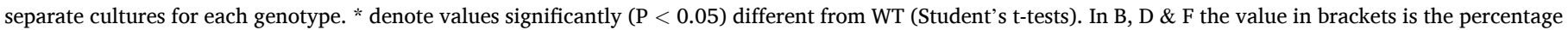
of $16: 0$ at the sn- 2 position.

was $\sim 62 \%$ in gsy1 1 -LPAAT2 (Fig. 3B). This is a similar level of 16:0 enrichment at sn-2 as we found for culture on glycerol (Fig. 2B and D).

The FA composition of TAG from WT and gsy1 $1-L P A A T 2$ cells grown on palm oil (Fig. 3C) was different from cells cultured on glycerol or glucose as sole carbon source (Figs. 2C and 3A). The levels of 16:0 increased significantly $(\mathrm{P}<0.05)$ to more than $26 \%$ and $16: 1$ and 18:0 reduced also to $\sim 4$ and $\sim 1 \%$, respectively. These changes are consistent with the FA composition of the palm oil substrate, which is rich in 16:0, 18:1 and 18:2, but contains little 16:1 and 18:0 (Supplementary Table 3). The percentage of total $16: 0$ at the sn-2 position was only $\sim 1 \%$ in WT, but it increased to $\sim 62 \%$ in gsy $1 \Delta-L P A A T 2$ (Fig. 3D), which is similar with the level of 16:0 enrichment at sn-2 obtained when this strain was cultured on glycerol or glucose (Figs. 2B and 3B). However, total $16: 0$ content in TAG is higher, while $18: 0$ at the $s n-1 / 3$ positions is also decreased, when gsy1 $1 \triangle$-LPAAT2 cells are cultured on palm oil versus either glucose or glycerol, because the total level of 18:0 in TAG is lowered and $18: 0$ is predominantly esterified at the $s n-1 / 3$ positions (Fig. 3C, Supplementary Table 2).

We also cultured WT and gsy1 $\triangle-L P A A T 2$ cells on a mixture of glucose and palm oil and found that the FA composition of the TAG was similar to culture on palm oil alone, with significantly $(\mathrm{P}<0.05)$ elevated levels of 16:0 ( $\sim 24 \%)$ and lowered levels of 16:1 and 18:0 ( $\sim 3 \%$ and $\sim 1 \%)$ (Fig. 3E) versus culture on glycerol or glucose (Figs. $2 \mathrm{C}$ and $3 \mathrm{~A}$ ). The percentage of total $16: 0$ at the sn-2 position was $\sim 1 \%$ in WT and increased to $\sim 69 \%$ in gsy $1 \Delta$-LPAAT2 (Fig. 3F). The enrichment of 16:0 at sn-2 when gsy1 $\triangle-L P A A T 2$ cells were cultured on a mixture of glucose and palm oil (Fig. $3 F$ ) was therefore significantly $(P<0.05)$ higher than when the cells were cultured on glycerol, glucose, or palm oil individually (Figs. 2B and 3B and D). As with culture on palm oil (Fig. 3C), the reduction in 18:0 content also provided lower 18:0 levels at sn-1/3, and there was a reduction in 16:1 (Fig. 3F).
3.3. Culture on palm oil or a mixture of glucose and palm oil increases lipid titre

To determine whether LPAAT2 expression affects Y. lipolytica cell biomass and lipid production in shake flask culture in nitrogen limited media we grew WT and gsy $1 \triangle$-LPAAT2 on glycerol, glucose, palm oil and a mixture of glucose and palm oil. After $72 \mathrm{~h}$ of culture on these carbon sources the cells had reached stationary phase (Bhutada et al., 2017). On glycerol or glucose, WT cell biomass reached $\sim 4 \mathrm{~g} \mathrm{~L}^{-1}$ (Table 2), whereas a significantly $(\mathrm{P}<0.05)$ higher biomass of $\sim 6 \mathrm{~g} \mathrm{~L}^{-1}$ was achieved on palm oil and on glucose plus palm oil (Table 2). gsy1 $\Delta-L$ $P A A T 2$ biomass was significantly $(\mathrm{P}<0.05)$ reduced when cells were cultured on glycerol and glucose but remained around the same as WT on palm oil or glucose plus palm oil (Table 2). The lipid content as a percentage of cell dry weight (\% CDW) of gsy1 1 -LPAAT2 cells grown on glycerol or glucose was significantly $(\mathrm{P}<0.05)$ higher than WT (Table 2). This increase is likely attributable to disruption of the GSY1 locus, rather than to LPAAT2 expression. GSY1 disruption is known to block glycogen synthesis and enhance lipid content (Bhutada et al., 2017). A comparison of gsy $1 \Delta$ and gsy $1 \Delta-L P A A T 2$ cells grown on nitrogen limited media with glucose showed that gsy1 $\Delta$ has a slightly higher lipid content $(18.2 \pm 0.3 \%$ versus $17.0 \pm 0.2 \%(\mathrm{n}=3)$ ). The biomass of gsy $1 \Delta$ and gsy $1 \Delta-L P A A T 2$ cells grown on glucose were $3.6 \pm 0.1$ and 3.7 $\pm 0.1 \mathrm{~g} \mathrm{~L}^{-1}(\mathrm{n}=3)$ respectively, which are not significantly different values $(\mathrm{P}>0.05)$ and this suggests that LPAAT2 expression is not detrimental to growth on this carbon source. The lipid content of WT and gsy1 $1-L P A A T 2$ cells grown on palm oil and on glucose plus palm oil was similar (in the range of $42-48 \%$ ) (Table 2). These values are more than double those obtained from culture on glycerol or glucose as a sole carbon source (Table 2). The combined gains in cell biomass and lipid content associated with culture on palm oil or on glucose plus palm oil 
Table 2

Cell biomass and lipid content of Y. lipolytica WT and gsy1 $1-L P A A T 2$ strains cultured on $20 \mathrm{~g} \mathrm{~L}^{-1}$ glycerol, glucose, or palm oil or on $10 \mathrm{~g} \mathrm{~L}^{-1}$ glucose $+10 \mathrm{~g}$ $\mathrm{L}^{-1}$ palm oil in nitrogen-limited media. The values are means \pm SD of measurements made on three separate cultures for each genotype. * denote values significantly $(\mathrm{P}<0.05)$ different from WT (Student's t-tests) and ${ }^{\#}$ from glycerol (ANOVA + Tukey HSD test).

\begin{tabular}{|c|c|c|c|c|}
\hline Strain & Substrate & $\begin{array}{l}\text { Cell biomass ( } \mathrm{g} \\
\mathrm{L}^{-1} \text { ) }\end{array}$ & $\begin{array}{l}\text { Lipid content (\% } \\
\text { of CDW) }\end{array}$ & $\begin{array}{l}\text { Lipid titre (g } \\
\mathrm{L}^{-1} \text { ) }\end{array}$ \\
\hline $\begin{array}{l}\text { WT } \\
\text { gsy1 } 1 \text { - } \\
\quad \text { LPAAT2 }\end{array}$ & Glycerol & $\begin{array}{l}4.0 \pm 0.1 \\
3.3 \pm 0.2^{*}\end{array}$ & $\begin{array}{l}11.5 \pm 0.5 \\
21.5 \pm 0.9 *\end{array}$ & $\begin{array}{l}0.5 \pm 0.1 \\
0.7 \pm 0.1 *\end{array}$ \\
\hline $\begin{array}{l}\text { WT } \\
\text { gsy1 } 1 \Delta- \\
\text { LPAAT2 }\end{array}$ & Glucose & $\begin{array}{l}4.0 \pm 0.1 \\
3.7 \pm 0.1^{* \#}\end{array}$ & $\begin{array}{l}10.1 \pm 1.8 \\
17.0 \pm 1.7 \text { *\# }\end{array}$ & $\begin{array}{l}0.4 \pm 0.1^{\#} \\
0.6 \pm 0.1^{* \#}\end{array}$ \\
\hline $\begin{array}{l}\text { WT } \\
\text { gsy1 } 1 \Delta- \\
\quad L P A A T 2\end{array}$ & Palm oil & $\begin{array}{l}6.3 \pm 1.1^{\#} \\
6.7 \pm 1.3^{* \#}\end{array}$ & $\begin{array}{l}48.0 \pm 6.5^{\#} \\
46.6 \pm 0.4^{\#}\end{array}$ & $\begin{array}{l}3.1 \pm 0.8^{\#} \\
3.1 \pm 0.5^{\#}\end{array}$ \\
\hline WT & $\begin{array}{l}\text { Glucose } \\
+\end{array}$ & $5.9 \pm 0.2^{\#}$ & $42.4 \pm 2.2^{\#}$ & $2.5 \pm 0.1^{\#}$ \\
\hline $\begin{array}{l}\text { gsy14- } \\
\text { LPAAT2 }\end{array}$ & Palm oil & $5.9 \pm 1.3^{\#}$ & 46.8. $\pm 2.1^{*}$ & $2.8 \pm 0.5^{\#}$ \\
\hline
\end{tabular}

mean that the lipid titre $\left(\sim 3 \mathrm{~g} \mathrm{~L}^{-1}\right)$ is four to five time higher than for culture on glycerol or glucose (Table 2 ).

\subsection{LPAAT2 expression also leads to $16: 0$ enrichment at the sn-2 position in an obese strain}

Several studies have showed that $Y$. lipolytica can be engineered to enhance TAG accumulation (Ledesma-Amaro and Nicaud, 2016). Lazar et al. (2014) created an 'obese' strain by overexpressing acyl-CoA: diacylglycerol acyltransferase (DGA2) and glycerol-3-phosphate dehydrogenase (GPD1) to enhance TAG biosynthesis (Beopoulos et al., 2012; Dulermo and Nicaud, 2011) and by deleting the six genes encoding acyl-coenzyme A oxidases (POX1-6) and the TAG lipase TGL4 to block peroxisomal FA $\beta$-oxidation and TAG hydrolysis, respectively (Beopoulos et al., 2008; Dulermo et al., 2013). To determine whether LPAAT2 expression also leads to 16:0 enrichment at the sn-2 position of TAG in this obese strain we integrated TEF1p-LPAAT2 at the GSY1 locus to create the strain obese-gsy1L-LPAAT2 (Table 1). Both the obese and obese-gsy1 $\triangle$-LPAAT2 strains were grown in triplicate in shake flask cultures in nitrogen-limited media with $20 \mathrm{~g} \mathrm{~L}^{-1}$ glucose or with $20 \mathrm{~g} \mathrm{~L}^{-1}$ glucose plus $10 \mathrm{~g} \mathrm{~L}^{-1}$ palm oil as the carbon source. Palm oil was not tested as a sole carbon source because the obese strain is deficient in FA $\beta$-oxidation (Beopoulos et al., 2008).

The total FA composition of TAG from obese cells grown on glucose as a sole carbon source for $72 \mathrm{~h}$ differs from that of WT, in that 16:0 is increase to $\sim 30 \%$ and $18: 2$ is reduced to $\sim 6 \%$ (Lazar et al., 2014). The percentage of 16:0 in TAG from obese-gsy1 $1-L P A A T 2$ cells is significantly $(\mathrm{P}<0.05)$ lower than in obese on both glucose (Fig. 4A) and on glucose plus palm oil (Fig. 4C). This is likely caused by disruption of GSY1 (Bhutada et al., 2017). Growth of obese on glucose plus palm oil also leads to a significantly $(\mathrm{P}<0.05)$ lower percentage of 16:1 and 18:0 (Fig. 4 C), as was observed in WT (Fig. $3 \mathrm{C}$ ). Less than $\sim 1 \%$ of the $16: 0$ in TAG is present at the sn-2 position in the obese strain grown either on glucose or on glucose plus palm oil (Fig. 4B and D). In TAG from obese-gsy1 1 -LPAAT2 cells, $\sim 52 \%$ and $\sim 62 \%$ of the $16: 0$ is at the sn- 2 position when the cells are grown on glucose and on glucose plus palm oil, respectively (Fig. 4B and D). Enrichment of 16:0 at the sn-2 position therefore appears to be a bit lower in obese-gsy1 $1-L P A A T 2$ than we measured in gsy1 $\triangle-L P A A T 2$, but culture on glucose plus palm oil also led to a significant $(\mathrm{P}<0.05)$ increase verses glucose alone (Fig. 4B and $\mathrm{D}$ ).

Using glucose as sole carbon source, obese and obese-gsy1 1 -LPAAT2 cell biomass reached around $5.8 \mathrm{~g} \mathrm{~L}^{-1}$ after $72 \mathrm{~h}$, whereas a significantly $(\mathrm{P}<0.05)$ higher biomass of $\sim 7.5 \mathrm{~g} \mathrm{~L}^{-1}$ was achieved on glucose plus palm oil (Table 3). The lipid content, as a \% CDW, of obese and obesegsy1 $\triangle$-LPAAT2 cells grown on glucose was $\sim 41 \%$ and $\sim 46 \%$, respectively (Table 3). The increase in obese-gsy1 $1-L P A A T 2$ is likely attributable to the disruption of GSY1 (Bhutada et al., 2017). On glucose plus palm oil, obese and obese-gsy1 1 -LPAAT2 lipid content reached around $\sim 67 \%$ of CDW. The gains in cell biomass and lipid content associated with culture on glucose plus palm oil mean that the lipid titre $(\sim 5.3 \mathrm{~g}$ $\mathrm{L}^{-1}$ ) is nearly two times higher than for culture on glucose (Table 3 ). Lipid titre in obese-gsy1 $1 \Delta$-LPAAT2 was also substantially higher than in gsy1 $\triangle-L P A A T 2$, which is consistent with the fact that the obese strain was previously engineered to accumulate more lipid (Lazar et al., 2014).

Growth curve measurements performed on obese and obese-gsy14LPAAT2 shake flask cultures, showed that cell biomass increases most
Glucose

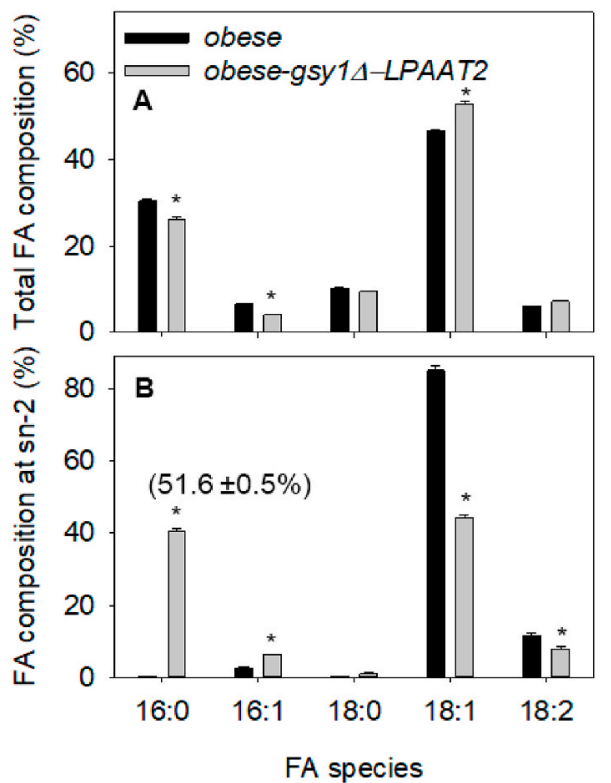

Glucose + Palm oil
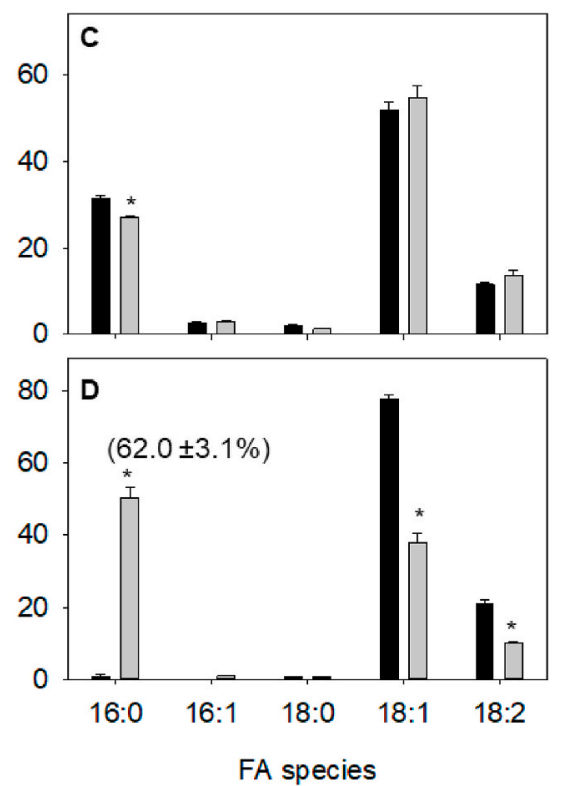

Fig. 4. Total (A \& C) and sn-2 (B \& D) FA composition of TAG from Y. lipolytica obese and obese-gsy14LPAAT2 strains cultured on $20 \mathrm{~g} \mathrm{~L}^{-1}$ glucose (A \& B), or a mixture of $20 \mathrm{~g} \mathrm{~L}^{-1}$ glucose and $10 \mathrm{~g} \mathrm{~L}^{-1}$ palm oil (C \& D) in nitrogen-limited media. The values are means \pm SD of measurements made on three separate cultures for each genotype. * denote values significantly $(\mathrm{P}<0.05)$ different from WT (Student's ttests). In B \& $\mathrm{D}$ the value in brackets is the percentage of $16: 0$ at the sn-2 position. 
Table 3

Cell biomass and lipid content of Y. lipolytica obese and obese-gsy1 $1 \Delta$-LPAAT2 strains cultured on $20 \mathrm{~g} \mathrm{~L}^{-1}$ glucose, or on $20 \mathrm{~g} \mathrm{~L}^{-1}$ glucose $+10 \mathrm{~g} \mathrm{~L}^{-1}$ palm oil in nitrogen-limited media. The values are means \pm SD of measurements made on three separate cultures for each genotype. * denote values significantly ( $\mathrm{P}<$ 0.05) different from obese and ${ }^{\#}$ from glucose (Student's t-tests).

\begin{tabular}{lllll}
\hline Strain & Substrate & $\begin{array}{l}\text { Cell biomass } \\
\left(\mathrm{g} \mathrm{L}^{-1}\right)\end{array}$ & $\begin{array}{l}\text { Lipid content }(\% \\
\text { of CDW) }\end{array}$ & $\begin{array}{l}\text { Lipid titre } \\
\left(\mathrm{g} \mathrm{L}^{-1}\right)\end{array}$ \\
\hline $\begin{array}{l}\text { obese } \\
\text { obese-gsy14- } \\
\text { LPAAT2 }\end{array}$ & Glucose & $5.8 \pm 0.3$ & $41.5 \pm 0.8$ & $2.4 \pm 0.2$ \\
& & $5.5 \pm 0.2^{*}$ & $45.9 \pm 1.7^{*}$ & $2.5 \pm 0.4$ \\
$\begin{array}{l}\text { obese } \\
\text { Gbese-gsy14- } \\
\text { LPAAT2 }\end{array}$ & $\begin{array}{l}\text { Glucose } \\
+\end{array}$ & $7.5 \pm 0.2^{\#}$ & $67.2 \pm 3.2^{\#}$ & $5.0 \pm 0.2^{\#}$ \\
\hline
\end{tabular}

rapidly within the first $24 \mathrm{~h}$ and is maximal at around $72 \mathrm{~h}$, for both strains (Fig. 5). Lipid titre also increases over this same period (Fig. 5). Both obese and obese-gsy1 $1-L P A A T 2$ consume glucose most rapidly within the first $48 \mathrm{~h}$ of cultivation and have metabolised more than $90 \%$ of the $20 \mathrm{~g} \mathrm{~L}^{-1}$ by $96 \mathrm{~h}$, regardless of whether they are provided glucose as sole carbon source, or glucose plus palm oil (Fig. 5). Where both glucose and palm oil are provided, the consumption of palm oil derived FA is delayed relative to that of glucose (Fig. 5B and D), as has been described previously in Y. lipolytica (Kamzolova et al., 2011). After 96 h, the loss of palm oil derived FA from the culture medium is approximately equivalent to the lipid content of the cell biomass for both obese and obese-gsy1 1 -LPAAT2 (Fig. 5B and D). Since both strains are deficient in $\beta$-oxidation (Beopoulos et al., 2008), this suggests that much of the TAG that accumulates in the cells is likely synthesised from imported FA. Citrate is a common co-product of $Y$. lipolytica fermentation under nitrogen limitation and it also accumulates over the time course of the shake flask cultivations (Fig. 5), as has been reported previously for obese cultured on glucose (Lazar et al., 2014). After $96 \mathrm{~h}$ of culture, the yield of biomass and of lipid from consumed glucose is 0.26 and $0.12 \mathrm{~g}$ $\mathrm{g}^{-1}$ and 0.26 and $0.13 \mathrm{~g} \mathrm{~g}^{-1}$ for obese and obese-gsy1L-LPAAT2 respectively (calculated from Fig. 5). These values are similar with those reported by Lazar et al. (2014) for obese. On glucose plus palm oil, the yield of biomass and of lipid is 0.26 and $0.17 \mathrm{~g} \mathrm{~g}^{-1}$ and 0.25 and $0.17 \mathrm{~g}$ $\mathrm{g}^{-1}$ for obese and obese-gsy1 $1 \Delta-L P A A T 2$ respectively. The higher lipid yield on glucose plus palm oil is consistent with the contribution of direct FA uptake and storage. Finally, batch culture of obese-gsy1 $\triangle$-LPAAT2 with glucose plus palm oil at $0.5 \mathrm{~L}$ scale in a bioreactor resulted in similar biomass production, lipid content and FA composition (Supplementary Tables 4 and 5) to that observed in shake flask cultures (Figs. 5 and 4).

\section{Discussion}

In this study we show that $Y$. lipolytica cells, that normally exclude 16:0 from the sn-2 position in their TAG (Thorpe and Ratledge 1972), can be engineered to incorporate more than $60 \%$ of the total $16: 0$ at the sn-2 position by expressing a LPAT with a preference of 16:0-CoA. The most effective LPAT we tested is LPAAT2 from the alga Chlamydomonas reinhardtii (Kim et al., 2018). The change in TAG stereoisomeric structure, combined with the native FA composition found in $Y$. lipolytica cells cultured on glycerol or glucose, produces fat that is similar to human milk, in terms of the major molecular species of FAs $(16: 0,18: 1$ and 18:2), and thus has the potential to be used as a HMFS ( $\beta$-palmitate) ingredient in infant formulas. Furthermore, we found that both the FA composition and yield of lipid that is produced by wildtype and a lipid overproducing obese $Y$. lipolytica strain (Lazar et al., 2014) expressing LPAAT2 can be improved, without further metabolic engineering, by culture on palm oil or on a mixture of glucose plus palm oil. Unexpectedly the combination of glucose plus palm oil also led to an increase in the percentage of $16: 0$ in the sn-2 position up to $\sim 70 \%$, which is similar to values reported for human milk fat (Breckenridge et al., 1969; Giuffrida et al., 2019). The reason why this mixture of substrates leads to a greater enrichment of $16: 0$ at sn-2 is unclear but combinations can influence the regulation of metabolism (Fabiszewska et al., 2015; Lubuta
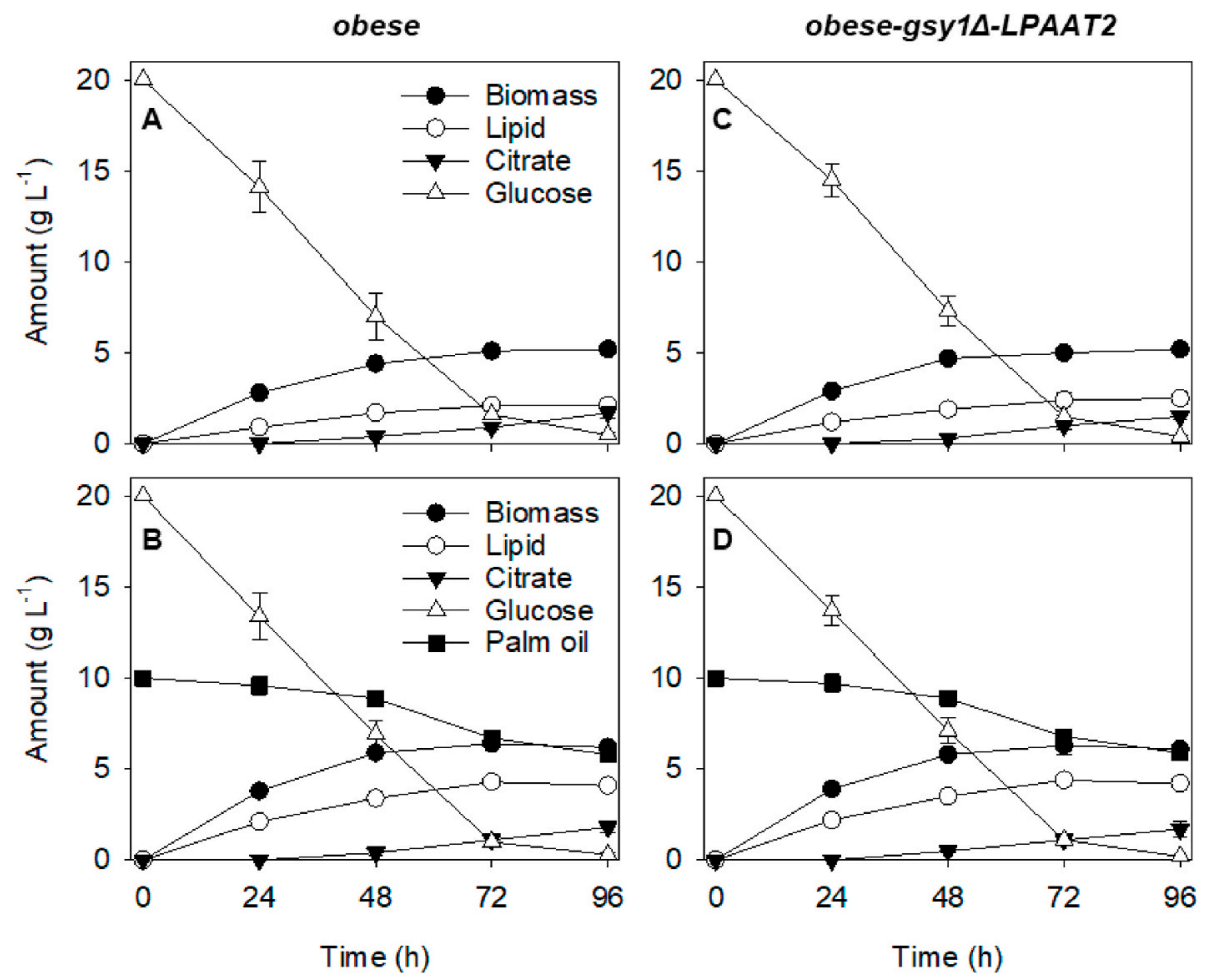

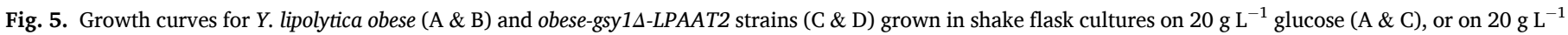

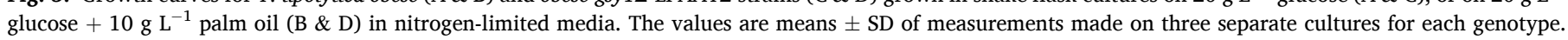


et al., 2019). Y. lipolytica uses glucose initially as a preferred carbon source when cultured on glucose plus 18:1, and then switches to use 18:1 once the glucose is depleted (Kamzolova et al., 2011).

$Y$. lipolytica is known to be flexible with regards to its carbon source and (as its name suggests) it can grow readily on a range of lipids as well as on glycerol, acetate, and certain sugars (Fickers et al., 2005: Spagnuolo et al., 2018). Its substrate range has also been successfully extended by GE (Spagnuolo et al., 2018), for example to include lignocellulosic materials (Niehus et al., 2018). The highest productivity in terms of lipids has been reported for $Y$. lipolytica strains with engineered cytosolic redox metabolism grown in nitrogen-limited glucose-fed batch culture, where $1.2 \mathrm{~g}^{-1} \mathrm{~L}^{-1} \mathrm{~h}^{-1}$ has been achieved (Qiao et al., 2017). These strains could provide a platform for more efficient HMFS production. It is noteworthy that similar or higher lipid productivities have also been reported for Rhodosporidium toruloides, Rhodotorula glutinis and Lipomyces starkeyi yeasts cultivated on glucose (Karamerou et al., 2021). The Y. lipolytica strains used in this study have not been engineered to improve total FA composition, but modification of this trait is possible based on the findings of previous studies (Ledesma-Amaro and Nicaud, 2016; Tsakraklides et al., 2018). It is noteworthy that human milk fat also contains low levels of medium chain saturated FAs $(\sim 12 \%)$ and very long chain polyunsaturated FAs $(\sim 2 \%)$ that are both absent from Y. lipolytica (Carsanba et al., 2020; López-López et al., 2002). These FAs are incorporated into the fat phase of infant formulas from palm kernel or coconut oil and from algae or fish oils, respectively (Wei et al., 2019). However, both these classes of FA can also be produced in $Y$. lipolytica by metabolic engineering (Rutter et al., 2015; Xue et al., 2013). Further enrichment of 16:0 at the sn-2 position in TAG may also be achievable in Y. lipolytica by supressing native LPATs (e.g. SLC1) and/or by disrupting acyl editing activities that might subsequently remove 16:0, as we have shown previously in oilseeds (van Erp et al., 2019, 2021). Exclusion of 16:0 from the sn-1/3 positions might also be achievable by replacing other native acyltransferases. Tsakraklides et al. (2018) showed that 16:0 levels in $Y$. lipolytica TAG can be reduced by deleting the glycerol-3-phosphate acyltransferase SCT1 and by overexpressing a homologue from Arxula adeninivorans.

$Y$. lipolytica produces extracellular lipases that hydrolyze lipids, allowing the cells to take up the free FAs and $\beta$-oxidise them (Fickers et al., 2005). The FAs are also used for lipid synthesis and, when in excess, are incorporated into TAG (Fickers et al., 2005). The FA composition of the TAG therefore partially reflects that of the lipid substrate (Papanikolaou et al., 2003; Vasiliadoua et al., 2018a, b). The palm oil that we used as a substrate in this study has a FA composition that differs from $Y$. lipolytica, leading to the synthesis of TAG that is closer to a HMFS. Specifically, the palm oil contributes a higher 16:0 content and a lower 18:0 and 16:1 content in $Y$. lipolytica TAG. Y. lipolytica also discriminates against uptake of exogenous 18:0 but selectively incorporates it into TAG (Papanikolaou and Aggelis 2003). Human milk fat contains both 16:1 and 18:0 (López-López et al., 2002) but they are less abundant than in wildtype (W29) Y. lipolytica cultured on glycerol or glucose. 18:0 is also mainly esterified to the sn-1/3 positions in Y. lipolytica TAG; even in cells expressing LPAAT2 or AGPAT1. These LPATs either exhibit fatty acyl chain length specificity that prohibits them from incorporating 18:0 into the sn-2 position of glycerolipids or they have comparatively little 18:0-CoA substrate. It is noteworthy that although 18:0 is a minor FA in human milk fat it is located in the sn-1/3 positions, unlike 16:0 (López-López et al., 2002). However, it is likely desirable to exclude $18: 0$ from the sn-1/3 positions of TAG used as a HMFS, for the same reason as 16:0, because when either of these long chain saturated FAs are released by sn- $1 / 3$ regioselective lipases in the infant gut they can form calcium soaps and aggregate, reducing lipid and calcium absorption (Innis 2011; Béghin et al., 2018).

It is known that when wildtype and lipid overproducing $Y$. lipolytica strains are cultured on lipids or on lipid and sugar mixtures the cells can achieve a higher lipid content (\% CDW) than when the cells are cultured on sugars alone (Beopoulos et al., 2008). Papanikolaou et al. (2003) investigated the capacity of a wildtype $Y$. lipolytica strain to produce cocoa butter equivalent (another commercially important class of structured lipid) using animal stearin, and reported that culture on stearin plus glycerol yielded the highest titre of $\sim 3.4 \mathrm{~g} \mathrm{~L}^{-1}$, which is similar to the titre we obtained through shake flask culture of gsy $1 \Delta-L$ PAAT2 on palm oil or on glucose plus palm oil. The lipid content we measured $(42-48 \%$ of $C D W)$, is also similar with that reported by Papanikolaou et al. (2003). However, it is higher than the values reported by Vasiliadoua et al. (2018a, b) who also used palm oil as a sole carbon source. In the obese genetic background cultured on glucose as sole carbon source, lipid titre and lipid content (\% CDW) are increased (Lazar et al., 2014). We found that this is also the case for culture on glucose plus palm oil. It is noteworthy that all the genetic modifications introduced into the obese background are designed to increase TAG accumulation, rather than directly targeting FA synthesis (Lazar et al., 2014). Integration of TEFp-LPAAT2 at the GSY1 locus also disrupts glycogen synthesis, which results in higher TAG content (Bhutada et al., 2017). The obese-gsy1L-LPAAT2 strain is therefore appropriately adapted to enhance lipid production using a lipid substrate.

Some mammals (e.g. H. sapiens), algae (e.g. Nannochloropsis oceanica and C. reinhardtii) and bacteria (e.g. Rhodococcus opacus) can incorporate 16:0 into the sn-2 position of their TAG (Breckenridge et al., 1969; Nobusawa et al., 2017; Kim et al., 2018; Wältermann et al., 2000). The algae and bacteria that are known to do this have FA compositions that are rather dissimilar to human milk fat. $R$. opacus for instance produces a high percentage of odd chain length FAs when cultured on glucose or glycerol (Kurosawa et al., 2010, 2015). However, R. opacus is lipolytic and the FA composition of its TAG can also reflect that of its lipid substrate (Alvarez et al., 1996). Zang et al. (2020a, b, c) have recently showed that HMFS can be made by culturing a wildtype $R$. opacus strain on mixtures of vegetable, animal and algal oils that have been chemically inter-esterified or on mixtures of FA ethyl esters. However, the lipid titre they report in shake flask culture under nitrogen limitation (Zhang et al., 2020a, b, c) are around a half and a quarter of the values we report here using an engineered $Y$. lipolytica strain grown on glucose or on glucose plus palm oil, respectively.

There are several issues to consider when using lipid (as opposed to sugar) substrates for microbial TAG production. For example, substrates that are immiscible in water such as lipids require emulsification and may therefore be harder to work with in large-scale aerobic fermenters. More of the neutral lipid that accumulates in the cells can also be in the form of sterol esters and FAs (Athenstaedt et al., 2006; Beopoulos et al., 2008). Vegetable oils (and their chemically modified derivatives) are also more expensive substrates than sugars (weight for weight), but they do have a higher energy density and the carbon conversion efficiency of converting lipids to lipids is greater, theoretically. Ultimately, a thorough technoeconomic analysis is required to determine what the lowest cost microbial fermentation process would likely be for HMFS production. However, for heterotrophic growth of oleaginous yeast on sugar substrates, many analyses have already been made that may be applicable, owing to interest in microbial production of similar lipids such as palm oil substitute (Karamerou et al., 2021). Karamerou et al. (2021) recently estimated that the lowest selling cost it may be theoretically possible to reach is $\sim \$ 1.2 \mathrm{~kg}^{-1}$ for a production scale of $48 \mathrm{k}$ tonnes $\mathrm{yr}^{-1}$. Estimates of current costs are higher; ranging from $\$ 1.7$ to $\$ 5.9 \mathrm{~kg}^{-1}$ (Koutinas et al., 2014; Braunwald et al., 2016; Parsons et al., 2019). Market research suggests that HMFS, made using enzyme-catalysis (Ferreira-Dias and Tecelão, 2014), cost more than $\sim \$ 6 \mathrm{~kg}^{-1}$ and the production scale is around $50 \mathrm{k}$ tonnes $\mathrm{yr}^{-1}$. Finally, on environmental and sustainability grounds, it may ultimately be considered preferable to use sugars, rather than vegetable oils (and palm oil in particular), as substrates for HMFS production.

\section{Authorship contributions}

P.J.E. conceived the research and supervised the experiments; G.B. 
designed and performed the strain engineering and shake flask experiments and analysed data, with assistance from P.J.E. R.L-A. provided materials and technical advice on cloning, transformation and bioreactor experiments. P.H. performed the bioreactor experiments and G.M. and R.K.B. carried out lipid analysis. P.J.E. wrote the article with contributions of all the authors.

\section{Funding}

This work was funded by the UK Biotechnology and Biological Sciences Research Council through grants BB/P012663/1 and BB/ T013176/1.

\section{Declaration of competing interest}

The authors declare there is no conflict of interest.

\section{Acknowledgments}

We wish to thank Dr Harrie van Erp for providing training in lipid analysis methods and Prof. Klaus Natter for providing materials. R.K.B. also acknowledges support from the Rothamsted International and DSTINSPIRE Faculty fellowship schemes.

\section{Appendix A. Supplementary data}

Supplementary data to this article can be found online at https://doi. org/10.1016/j.mec.2022.e00192.

\section{References}

Agarwal, A.K., Sukumaran, S., Cortés, V.A., Tunison, K., Mizrachi, D., Sankella, S., Gerard, R.D., Horton, J.D., Garg, A., 2011. Human 1-acylglycerol-3-phosphate Oacyltransferase isoforms 1 and 2: biochemical characterization and inability to rescue hepatic steatosis in Agpat2(-/-) gene lipodystrophic mice. J. Biol. Chem. 286, 37676-37691.

Alvarez, H.M., Mayer, F., Fabritius, D., Steinbüchel, A., 1996. Formation of intracytoplasmic lipid inclusions by Rhodococcus opacus strain PD630. Arch. Microbiol. 165, 377-386.

Athenstaedt, K., Jolivet, P., Boulard, C., Zivy, M., Negroni, L., Nicaud, J.-M., Chardot, C., 2006. Lipid particle composition of the yeast Yarrowia lipolytica depends on the carbon source. Proteomics 6, 1450-1459.

Ayciriex, S., Le Guédard, M., Camougrand, N., Velours, G., Schoene, M., Leone, S., Wattelet-Boyer, V., Dupuy, J.-W., Shevchenko, A., Schmitter, J.-M., Lessire, R., Bessoule, J.-J., Testet, E., 2012. YPR139c/LOA1 encodes a novel lysophosphatidic acid acyltransferase associated with lipid droplets and involved in TAG homeostasis. Mol. Biol. Cell 23, 233-246.

Barth, G., Gaillardin, C., 1996. Yarrowia Lipolytica. Nonconventional Yeasts in Biotechnology: A Handbook. Springer Berlin Heidelberg, Berlin, Heidelberg, pp. 313-388.

Bates, P.D., Fatihi, A., Snapp, A.R., Carlsson, A.S., Browse, J., Lu, C., 2012. Acyl editing and headgroup exchange are the major mechanisms that direct polyunsaturated fatty acid flux into triacylglycerols. Plant Physiol. 160, 1530-1539.

Béghin, L., Marchandise, X., Liend, E., Bricoute, M., Bernete, J.-P., Lienhardte, J.-F., Jeannerote, F., Menete, V., Requillarte, J.-C., Marx, J., et al., 2018. Growth, stool consistency and bone mineral content in healthy term infants fed sn-2-palmitateenriched starter infant formula: a randomized, double-blind, multicentre clinical trial. Clin. Nutr. 38, 1023-1030.

Benghezal, M., Roubaty, C., Veepuri, V., Knudsen, J., Conzelmann, A., 2007. SLC1 and SLC4 encode partially redundant acyl-coenzyme A 1-acylglycerol-3-phosphate Oacyltransferases of budding yeast. J. Biol. Chem. 282, 30845-30855.

Beopoulos, A., Haddouche, R., Kabran, P., Dulermo, T., Chardot, T., Nicaud, J.M., 2012 Identification and characterization of DGA2, an acyltransferase of the DGAT1 acylCoA:diacylglycerol acyltransferase family in the oleaginous yeast Yarrowia lipolytica. New insights into the storage lipid metabolism of oleaginous yeasts. Appl. Microbiol. Biotechnol. 93, 1523-1537.

Beopoulos, A., Mrozova, Z., Thevenieau, F., Le Dall, M.-T., Hapala, I., Papanikolaou, S., Chardot, T., Nicaudet, J.-M., 2008. Control of lipid accumulation in the yeast Yarrowia lipolytica. Appl. Environ. Microbiol. 74, 7779-7789.

Bhutada, G., Kavšcek, M., Ledesma-Amaro, R., Thomas, S., Rechberger, G.N., Nicaud, J.M., Natter, K., 2017. Sugar versus fat: elimination of glycogen storage improves lipid accumulation in Yarrowia lipolytica. FEMS Yeast Res. 17, fox020.

Bourgis, F., Kader, J.-C., Barret, P., Renard, M., Robinson, D., Robinson, C., Delseny, M., Roscoe, T.J., 1999. A plastidial lysophosphatidic acid acyltransferase from oilseed rape. Plant Physiol. 120, 913-922.
Braunwald, T., French, W.T., Claupein, W., Graeff-Hönninger, S., 2016. Economic assessment of microbial biodiesel production using heterotrophic yeasts. Int. J. Green Energy 13, 274-282.

Breckenridge, W.C., Marai, L., Kuksis, A., 1969. Triglyceride structure of human milk fat. Can. J. Biochem. 47, 761-769.

Bredeweg, E.L., Pomraning, K.R., Dai, Z., Nielsen, J., Kerkhoven, E.J., Baker, S.E., 2017. A molecular genetic toolbox for Yarrowia lipolytica. Biotechnol. Biofuels 10, 2-22.

Brockerhoff, H., Yurkowsk, M., 1966. Stereospecific analyses of several vegetable fats. J. Lipid Res. 7, 62-64.

Carsanba, E., Papanikolaou, S., 3 Fickers, P., Erten, H., 2020. Lipids by Yarrowia lipolytica strains cultivated on glucose in batch cultures. Microorganisms 8, 1054.

Christie, W.W., Clapperton, J.L., 1982. Structures of the triglycerides of cows' milk, fortified milks (including. infant formulae), and human milk. Int J Dairy Technol 35, $22-24$.

Christie, W.W., Moore, J.H., 1970. A comparison of the structures of triglycerides from various pig tissues. Biochim. Biophys. Acta Mol. Cell Biol. Lipids 210, 46-56.

Christie, W.W., Nikolova-Damyanova, B., Laakso, P., Herslof, B., 1991. Stereospecific analysis of triacyl-sn-glycerols via resolution of diastereomeric diacylglycerol derivatives by high-performance liquid chromatography on silica. J. Am. Oil Chem. Soc. 68, 695-701.

Dulermo, T., Tréton, B., Beopoulos, A., Kabran Gnankon, A.P., Haddouche, R., Nicaud, J. M., 2013. Characterization of the two intracellular lipases of $Y$. lipolytica encoded by TGL3 and TGL4 genes: new insights into the role of intracellular lipases and lipid body organisation. Biochim. Biophys. Acta 1831, 1486-1495.

Curran, K.A., Morse, N.J., Markham, K.A., Wagman, A.M., Gupta, A., Alper, H.S., 2015. Short synthetic terminators for improved heterologous gene expression in yeast. ACS Synth. Biol. 17, 824-832.

Dulermo, T., Nicaud, J.M., 2011. Involvement of the G3P shuttle and $\beta$-oxidation pathway in the control of TAG synthesis and lipid accumulation in Yarrowia lipolytica. Metab. Eng. 13, 482-491.

Fabiszewska, A.U., Kotyrba, D., Nowak, D., 2015. Assortment of carbon sources in medium for Yarrowia lipolytica lipase production: a statistical approach. Ann. Microbiol. 65, 1495-1503.

Ferreira-Dias, S., Tecelão, C., 2014. Human milk fat substitutes: advances and constraints of enzyme-catalyzed production. Lipid Technol. 26, 183-186.

Fickers, P., Benetti, P.-H., Waché, Y., Marty, A., Mauersberger, S., Smit, M.S., Nicaud, J.M., 2005. Hydrophobic substrate utilisation by the yeast Yarrowia lipolytica, and its potential applications. FEMS Yeast Res. 5, 527-543.

Gibson, D.G., Young, L., Chuang, R.-Y., Venter, J.C., Hutchison, C.A., Smith, H.O., 2009. Enzymatic assembly of DNA molecules up to several hundred kilobases. Nat. Methods 6, 343-345.

Giuffrida, F., Marmet, C., Tavazzi, I., Fontannaz, P., Sauser, J., Lee, L.Y., Destaillats, F., 2019. Quantification of 1,3-olein-2-palmitin (OPO) and palmitic acid in sn-2 position of triacylglycerols in human milk by liquid chromatography coupled with mass spectrometry. Molecules 24, 22.

Groenewald, M., Boekhout, T., Neuvéglise, C., Gaillardin, C., van Dijck, P.W.M., Wysset, M., 2014. Yarrowia lipolytica: safety assessment of an oleaginous yeast with a great industrial potential. Crit. Rev. Microbiol. 40, 187-206.

Hanscho, M., Ruckerbauer, D.E., Chauhan, N., Hofbauer, H.F., Krahulec, S., Nidetzky, B., Kohlwein, S.D., Zanghellini, J., Natter, K., 2012. Nutritional requirements of the BY series of Saccharomyces cerevisiae strains for optimum growth. FEMS Yeast Res. 12, 796-808.

Hong, K.K., Nielsen, J., 2013. Adaptively evolved yeast mutants on galactose show tradeoffs in carbon utilization on glucose. Metab. Eng. 16, 78-86.

Innis, S.M., 2011. Dietary triacylglycerol structure and its role in infant nutrition. Ady Nutr 2, 275-283.

Jain, S., Stanford, N., Bhagwat, N., Seiler, B., Costanzo, M., Boone, C., Oelkers, P., 2007. Identification of a novel lysophospholipid acyltransferase in Saccharomyces cerevisiae. J. Biol. Chem. 282, 30562-30569.

Kamzolova, S.V., Lunina, J.N., Morgunov, I.G., 2011. Biochemistry of citric acid production from rapeseed oil by Yarrowia lipolytica yeast. JAOCS (J. Am. Oil Chem. Soc.) $88,1965-1976$.

Karamerou, E.E., Parsons, S., McManus, M.C., Chuck, C.J., 2021. Using techno-economic modelling to determine the minimum cost possible for a microbial palm oil substitute. Biotechnol. Biofuels 14, 57.

Kim, H.U., Li, Y., Huang, A.H., 2005. Ubiquitous and endoplasmic reticulum-located lysophosphatidyl acyltransferase, LPAT2, is essential for female but not male gametophyte development in Arabidopsis. Plant Cell 17, 1073-1089.

Kim, Y., Terng, E.L., Riekhof, W.R., Cahoon, E.B., Cerutti, H., 2018. Endoplasmic reticulum acyltransferase with prokaryotic substrate preference contributes to triacylglycerol assembly in Chlamydomonas. Proc. Natl. Acad. Sci. U.S.A. 115, 1652-1657.

Klug, L., Daum, G., 2014. Yeast lipid metabolism at a glance. FEMS Yeast Res. 14, 369-388.

Koutinas, A.A., Chatzifragkou, A., Kopsahelis, N., Papanikolaou, S., Kookos, I.K., 2014. Design and techno-economic evaluation of microbial oil production as a renewable resource for biodiesel and oleochemical production. Fuel 116, 566-577.

Kurosawa, K., Boccazzi, P., de Almeida, N.M., Sinskey, A.J., 2010. High-cell-density batch fermentation of Rhodococcus opacus PD630 using a high glucose concentration for triacylglycerol production. J. Biotechnol. 147, 212-218.

Kurosawa, K., Radek, A., Plassmeier, J.K., Sinskey, A.J., 2015. Improved glycerol utilization by a triacylglycerol-producing Rhodococcus opacus strain for renewable fuels. Biotechnol. Biofuels 8, 31 .

Lazar, Z., Dulerma, T., Neuvéglise, C., Crutz-Le Coq, A.-M., Nicaud, J.-M., 2014. Hexokinase-A limiting factor in lipid production from fructose in Yarrowia lipolytica. Metab. Eng. 26, 89-99. 
Le Dall, M.-T., Nicaud, J.-M., Gaillardin, C., 1994. Multiple-copy integration in the yeast Yarrowia lipolytica. Curr. Genet. 26, 38-44.

Ledesma-Amaro, R., Nicaud, J.-M., 2016. Yarrowia lipolytica as a biotechnological chassis to produce usual and unusual fatty acids. Prog. Lipid Res. 61, 40-50.

López-López, A., López-Sabater, M.C., Campoy-Folgoso, C., Rivero-Urgell, M., CastelloteBargalló, A.I., 2002. Fatty acid and sn-2 fatty acid composition in human milk from Granada (Spain) and in infant formulas. Eur. J. Clin. Nutr. 56, 1242-1254.

Lu, C., Xin, Z., Ren, Z., Miquel, M., Browse, J., 2009. An enzyme regulating triacylglycerol composition is encoded by the ROD1 gene of Arabidopsis. Proc. Natl. Acad. Sci. U.S.A. 106, 18837-18842.

Lubuta, P., Workman, M., Kerkhoven, E.J., Workman, C.T., 2019. Investigating the influence of glycerol on the utilization of glucose in Yarrowia lipolytica using RNASeq-based transcriptomics. G3 (Bethesda) 9, 4059-4071.

Miquel, M., Browse, J., 1992. Arabidopsis mutants deficient in polyunsaturated fatty acid synthesis. Biochemical and genetic characterization of a plant oleoylphosphatidylcholine desaturase. J. Biol. Chem. 267, 1502-1509.

Niehus, X., Crutz-Le Coq, A.-M., Sandoval, G., Nicaud, J.-M., Ledesma-Amaro, R., 2018. Engineering Yarrowia lipolytica to enhance lipid production from lignocellulosic materials. Biotechnol. Biofuels 11, 11.

Nobusawa, T., Hori, K., Mori, H., Kurokawa, K., Ohta, H., 2017. Differently localized lysophosphatidic acid acyltransferases crucial for triacylglycerol biosynthesis in the oleaginous alga Nannochloropsis. Plant J. 90, 547-559.

Ohlrogge, J., Browse, J., 1995. Lipid biosynthesis. Plant Cell 7, 957-970.

Papanikolaou, S., Aggelis, G., 2003. Selective uptake of fatty acids by the yeast Yarrowia lipolytica. Eur. J. Lipid Sci. Technol. 105, 651-655.

Papanikolaou, S., Muniglia, L., Chevalot, I., Aggelis, G., Marc, I., 2003. Accumulation of a cocoa-butter-like lipid by Yarrowia lipolytica cultivated on agro-industrial residues. Curr. Microbiol. 46, 124-130.

Parsons, S., Abeln, F., McManus, M.C., Chuck, C.J., 2019. Techno-economic analysis (TEA) of microbial oil production from waste resources as part of a biorefinery concept: assessment at multiple scales under uncertainty. J. Chem. Technol. Biotechnol. 94, 701-711.

Qiao, K., Wasylenko, T.M., Zhou, K., Xu, P., Stephanopoulos, G., 2017. Lipid production in Yarrowia lipolytica is maximized by engineering cytosolic redox metabolism. Nat. Biotechnol. 35, 173-177.

Rutter, C.D., Zhang, S., Rao, C.V., 2015. Engineering Yarrowia lipolytica for production of medium-chain fatty acids. Appl. Microbiol. Biotechnol. 99, 7359-7368.

Sambrook, J., Russell, D.W., 2001. In: Molecular Cloning: A Laboratory Manual, third ed. Cold Spring Harbor Laboratory Press, Cold Spring Harbour, NY, USA, pp. 1-3.

Spagnuolo, M., Hussain, M.S., Gambill, L., Blenner, M., 2018. Alternative substrate metabolism in Yarrowia lipolytica. Front. Microbiol. 9, 1077.

Stymne, S., Stobart, A.K., 1984. Evidence for the reversibility of the acyl-CoA: lysophosphatidylcholine acyltransferase in microsomal preparations from developing safflower (Carthamus tinctorius L.) cotyledons and rat liver. Biochem. J. 223, 305-314.

Thorpe, R.F., Ratledge, C., 1972. Fatty acid distribution in triglycerides of yeasts grown on glucose or n-alkanes. Microbiology 72, 151.

Turck, D., et al., 2019. Safety of Yarrowia lipolytica yeast biomass as a novel food pursuant to Regulation (EU) 2015/2283. EFSA J. 17, e05594.

Tsakraklides, V., et al., 2018. High-oleate yeast oil without polyunsaturated fatty acids. Biotechnol. Biofuels 11, 131.

van Erp, H., Bryant, F.M., Martin-Moreno, J., Michaelson, L.V., Bhutada, G., Eastmond, P.J., 2019. Engineering the stereoisomeric structure of seed oil to mimic human milk fat. Proc. Natl. Acad. Sci. U.S.A. 116, 20947-20952.

van Erp, H., Bryant, F.M., Martin-Moreno, J., Michaelson, L.V., Eastmond, P.J., 2021. Production of the infant formula ingredient 1,3-olein-2-palmitin in Arabidopsis thaliana seeds. Metab. Eng. 67, 67-74.

Vasiliadoua, I.A., Belloua, S., Daskalakia, A., Tomaszewska- Hetmana, L., Chatzikotoulaa, C., Kompotia, B., Papanikolaoub, S., Vayenascd, D., Pavloucd, S., Aggelis, G., 2018a. Biomodification of fats and oils and scenarios of adding value on renewable fatty materials through microbial fermentations: modelling and trials with Yarrowia lipolytica. J. Clean. Prod. 200, 1111-1129.

Vasiliadoua, I.A., Belloua, S., Daskalakia, A., Tomaszewska- Hetmana, L., Chatzikotoulaa, C., Kompotia, B., Papanikolaoub, S., Vayenascd, D., Pavloucd, S., Aggelis, G., 2018b. Data on cellular lipids of Yarrowia lipolytica grown on fatty substrates. Data Brief 21, 1037-1044.

Wältermann, M., Luftmann, H., Baumeister, D., Kalscheuer, R., Steinbüchelet, A., 2000. Rhodococcus opacus strain PD630 as a new source of high-value single-cell oil? Isolation and characterization of triacylglycerols and other storage lipids. Microbiology 146, 1143-1149.

Wang, L., Shen, W., Kazachkov, M., Chen, G., Chen, Q., Carlsson, A.S., Stymne, S., Weselake, R.J., Zou, J., 2012. Metabolic interactions between the Lands cycle and the Kennedy pathway of glycerolipid synthesis in Arabidopsis developing seeds. Plant Cell 24, 4652-4669.

Wei, W., Jin, Q., Wang, X., 2019. Human milk fat substitutes: past achievements and current trends. Prog. Lipid Res. 74, 69-86.

Xue, Z., et al., 2013. Production of omega-3 eicosapentaenoic acid by metabolic engineering of Yarrowia lipolytica. Nat. Biotechnol. 31, 734-740.

Zhang, L.-S., Chu, M.-Y., Zong, M.-H., Yang, J.-G., Lou, W.-Y., 2020a. Carbon source modify lipids composition of Rhodococcus opacus intended for infant formula. J. Biotechnol. 319, 8-14.

Zhang, L.-S., Chu, M.-Y., Zong, M.-H., Yang, J.-G., Lou, W.-Y., 2020b. Facile and green production of human milk fat substitute through Rhodococcus opacus fermentation. J. Agric. Food Chem. 68, 9368-9376.

Zhang, L.-S., Chu, M.-Y., Zong, M.-H., Yang, J.-G., Lou, W.-Y., 2020c. Efficient production of 1,3-dioleoyl-2-palmitoylglycerol through Rhodococcus opacus fermentation. J. Am. Oil Chem. Soc. 97, 851-860. 